\title{
X.500 Performance with Large Databases and OSInet $^{1}$
}

\author{
P.V.Rajeev ${ }^{2}$ S.V Raghavan ${ }^{3}$ \\ Department of Computer Science and Engineering \\ Indian Institute of Technology, Madras, India \\ rajeev\%hss@lando.hns.com \\ svr@iitm.ernet.in \\ Ashok K. Agrawala \\ Department of Computer Science, \\ University of Maryland, \\ College Park, MD USA. \\ agrawala@cs.umd.edu
}

\begin{abstract}
The X.500 directory provides a powerful mechanism for storing and retrieving information about objects of interest in the networking world. The rapid increase in the deployment of the directory has resulted in a very large amount of data in the global directory. Hence, the need arises for the development of a model which predicts the performance of the directory in an internet environment with large amount of data.

In this paper, we present a deterministic model for predicting the performance of X.500 directory operations. Actual measurements are carried out in a real life environment, with the directory having a large amount of data distributed across the internet. The model is validated by comparing the results of these actual measurements with the predicted values. The application of the model for the selection of DIT structure in a wide area network is explained.
\end{abstract}

\section{Keywords:}

X.500, Directory Services, Performance, Deterministic Modelling.

\footnotetext{
${ }^{1}$ Network Software developed at Indian Institute of Technology, Madras.

${ }^{2}$ Currently with Hughes Software Systems, New Delhi

${ }^{3}$ Further correspondence may please be addressed to Dr. S.V. Raghavan.
} 


\section{INTRODUCTION}

With the rapid expansion in the electronic communication, there is a growing need for an organized global information service. The X.500 directory provides such a powerful mechanism for storing and retrieving information about objects of interest in the networking world[CCITT (1988), Huitema (1989), Bumbulis et al.(1993)]. X.500 directory defines an architecture whereby the directory can be distributed over an arbitrary number of systems. Each system manages a portion of the global directory. Typically, in the X.500 directory, the information is distributed and managed across the network of many autonomous administrations. These systems are integrated into a single directory by a set of protocols. The directory stores information about objects and defines a hierarchical relationship between objects stored in the directory. The hierarchical structure reflects the national and organizational structure of the real world objects.

The X.500 directory was originally designed for use with X.400 message handling systems (MHS)[CCITT (1988)]. Message handling system uses directory for a variety of purposes including: retrieval of $O / R$ address when $O / R$ name is given, user friendly naming of users in the MHS, expansion of distribution lists, e-mail routing, etc. To provide these services to the MHS, the directory must store information about all e-mail users in the network. The number of e-mail users in the network itself will be of the order of millions. A recent survey on the usage of internet indicated a very high rate of increase in the number of users. A large amount of information has to be stored in the directory to cater to the needs of MHS.

Apart from MHS, dịrectory is now being used for a variety of other applications. For example directory is now used to store the topology and service related information about communication networks [Mánsfield et al.(1993), Hong et al.(1992a), Hong et al.(1992b)], to map object names to internal object identifiers in object based distributed operating systems [Rennesse et al.], to store bibliographic information [Barker (1992)], to store the information about ftp archives [Barker (1993)], and so on. Once all these applications start using the directory to store information, directory will be used extensively and an enormous amount of data will be stored in the directory.

The X.500 directory deployment in the internet is now progressing at a very fast pace. Presently, there are more than 450 Directory System Agents (DSAs) spanning 35 nations, covering a total of more than a million entries [Goodman (1992)]. The growth rate is found to be significant, with about 150 DSAs added every year since November 1990, with a proportional increase in the number of entries. With this rate of increase in the deployment of directory in the internet, the internet directory will soon be populated with enormous amount of information. The information about the e-mail users itself will be substantial. The internet directory is also used for storing information about various services available in the internet. Directory helps in giving an integrated view of the 
internet and the services available in the internet.

The concern of the designers is to estimate the critical database size and interconnection topologies among the DSAs and understand the implication of chaining and referral and guarantee response time whenever possible. This necessitates the study of the performance of the X.500 directory system, with special emphasis on large databases, wide area distribution and variable interconnections through different topologies.

In this paper, we present the performance measurements carried out in an internet environment with the directory distributed over two sites in the internet. A model for estimating the performance of the directory is also described.

The rest of the paper is organized as follows. Section 2 describes the X.500 and its implementation over OSInet. A deterministic model for X.500 is presented in the section 3. Section 4 validates the model with the help of actual measurements. The application of the model for the selection of DIT topology in a wide area network is explained in section 5 .

\section{X.500 OVER OSInet}

The X.500 directory used for the performance measurements presented in this paper, is implemented [Sriram (1991), Sriram et al.(1994), Raghavan et al.(1992)] over OSInet network kernel. OSInet [Raghavan (1990), Raghavan (1993a)] is a network protocol software which provides a subset of services defined in ISO-OSI reference model. OSInet has a network kernel which provides the basic networking support and user applications that run over the kernel. The kernel provides the services required for a reliable networking environment. OSInet follows the layered architecture of the ISO-OSI reference model. The interfaces to upper and lower layers are implemented using queues and the layer specific actions are realized through finite state machines. The OSInet architecture is shown in Figure 1a.

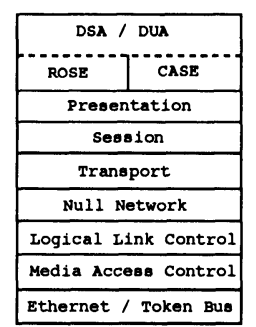

a. OsInet Architecture For LANg

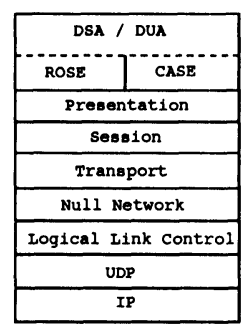

b. OsInet Architecture For Internet Experiments

Figure 1: The OSInet Architecture. 
The X.500 implementation makes use of ROSE and ACSE of OSInet. The binding and unbinding to $D S A s$ are done by using the ACSE. ROSE is used to invoke the directory operations. For the internet experiments the lower layers of OSInet stack is modified to include UDP as shown in Figure $1 \mathrm{~b}$.

The X.500 standard [CCITT (1988)] does not specify any database mechanism for storing the directory information. It is upto the implementor to choose an appropriate storage mechanism. In the case of OSInet X.500, B-Tree++ indexing mechanism is used [Comer (1979), Raghavan et al.(1993b)] for the database access. The tree structure of the directory is mapped onto the underlying file systems' directory structure. The knowledge information regarding the location of various naming contexts known to DSAs is stored in a sub-directory called tree directory, which is the working directory of a $D S A$. The information about an entry is stored as pairs <attribute type, attribute value> in the directory information files. The knowledge information file is indexed using the distinguished name as the key and the directory information file is indexed using the relative distinguished name (RDN) as the key.

\section{A DETERMINISTIC MODEL FOR X.500 DIRECTORY}

A deterministic model for distributed directory services allows one to investigate the effect of various design choices and mode of interaction between the DSAs on the cost of directory operations. Very few attempts have been made so far in the literature to quantify the performance of a distributed directory[Neufeld (1992)].

The major factors that affect the performance of the directory in a large internet environment are,

- the communication delay between the Directory User Agents (DUAs) and DSAs, and between $D S A s$,

- the database access time, and

- the name resolution time.

In this section we discuss a model for the performance estimation of the directory based on these factors.

Typically, a directory service consists of a number of DSAs distributed throughout the internet. In our model, we assume that the DSAs are always available and there are no communication link failures. A $D S A$ is run as a single process in the system.

The $D U A s$, or the clients of the directory services, may be a specific program, or it may be a part of another program which uses the directory for some lookup operation. For example, it may be a MHS UA which uses the directory to provide user friendly names to the MHS users. A $D U A$ need only know the address of its closest $D S A$. 
If a $D S A$ is unable to service a request, it may chain the request to another $D S A$ which may be able to answer the request. This may be repeated many times to resolve the name and to locate the requested entry. The $D S A$ may also return a referral back to the requested $D U A$ which in turn uses the referral to contact another $D S A$ whose address is returned in the referral. This $D S A$ can resolve the name if the requested object is present in its local database or can give a referral to another DSA which holds the entry or is more closer to the requested information. These modes of interactions are called chaining and referral respectively.

\subsection{Communication}

The roundtrip time between the $D U A$ and the $D S A_{i}$ is given by $C_{i} . C_{i}$ strongly depends on the sites at which the $D S A$ and the $D U A$ are executing. This value depends on the number of gateways between the $D S A$ and $D U A$ and also on the speed of the intermediate transmission links. We assume that the number bytes per packet for different operations are the same. Thus the different operations have the same roundtrip time. We also ignore the variations due to the network congestion. The round trip time between a $D S A_{i}$ and a $D S A_{j}$ is given by $C_{i j}$.

\subsection{Name resolution}

The $D S A$ resolves the name by scanning through its knowledge information base. If the requested object is located in locally held naming context, the $D S A$ goes down the tree and locates the requested object. If the $D S A$ finds that the requested object is not held locally, it finds out the address of the $D S A$ which stores the object or a $D S A$ which can give a more precise location of the $D S A$ which stores the object. Then it chains to the that $D S A$ or returns a referral back to the requested $D U A$. Let $N_{j}$ be the average amount of time required to perform the name resolution in the $D S A_{i}$.

\subsection{Database Access}

The second factor that affects the performance of the directory is the database access time. As we are storing enormous amount of data in the directory, the database access time will contribute to a significant portion of the response time as seen by the client. The database access time depends on two factors: the object accessed and the type of operation requested. Let the time required to perform the database operation be $D_{i}$ to perform an operation in the $D S A_{i}$. This is taken as an average amount of time taken to access different objects in the directory. 


\subsection{Other factors}

Another factor that affect the performance of the directoty is the protocol overhead due to the lower layers. The time spent in the protocol stack, $P_{i}$, is assumed to be constant for all objects and all operations. This will be true in most of the cases because the amount of data transferred by the directory operations will be almost the same. The protocol overhead depends mainly on the size of the PDUs transferred.

Partitioning of the name space also influences the performance of the directory. If enough cross references are stored in the $D S A s$, then the $D S A s$ will be able to resolve the name by contacting fewer number of $D S A$ s. Also by suitably selecting the higher level $D S A s$, the performance of the system can be improved.

\subsection{Scenarios}

Now, we proceed to find out the response time of the directory as seen by the user of the directory. We consider the following three different cases:

$i$ The local $D S A$ itself can answer the query.

ii The local DSA is unable to resolve the query and uses chaining mode of interaction.

iii The local $D S A$ is unable to resolve the query and uses referral.

We consider each of these three cases separately and find out the response time required in each case. In all these cases, we assume that the $D U A$ is already bound to its closest $D S A$ (primary $D S A$ ).

\subsection{Case I - The Simple Case}

In this case the interactions that are taking place is shown in the Figure 2. The DUA sends a request to the $D S A$ and $D S A$ reads its local database and responds to the query.

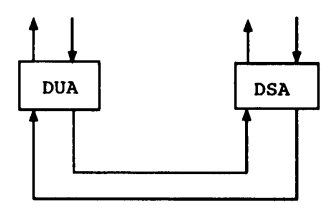

Figure 2: Single $D S A$, Single $D U A$

The response time as seen by the users of the $D U A$ for performing an operation can be given by:

Where,

$$
T=P_{c}+C_{1}+P_{1}+N_{1}+D_{1}
$$


$P_{c}=$ Protocol overhead in the client $(D U A)$.

$C_{1}=$ Round trip communication delay between the $D U A$ to $D S A_{1}$.

$P_{1}=$ Protocol overhead in the $D S A_{1}$ (including queuing delay).

$N_{1}=$ Name resolution time in $D S A_{1}$.

$D_{1}=$ Database access time in $D S A_{1}$.

\subsection{Case II - Chaining}

In this case, the primary $D S A, D S A_{1}$, decides to chain the request to a remote $D S A$ which can answer the query. The interactions that are taking place are shown in the Figure 3. The primary $D S A$ has to bind to the remote $D S A$ before invoking the remote operation on the second $D S A$. Thus the total time required to perform the operation includes the time required to bind to the remote $D S A+$ the time required to chain the request to the remote $D S A+$ the time required to merge the results obtained from the remote $D S A$ and send the result back to the $D U A$.

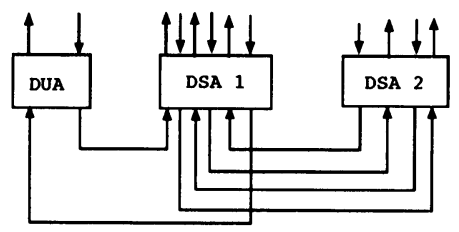

Figure 3: Multiple $D S A s$, Chaining mode of interaction

Thus the response time as seen by the user of the system is

$\mathrm{T}=$ time taken by the $D U A$ to send the request + time taken by the $D S A$ to perform the complete operation (include the time to bind to the second $D S A$ and to invoke remote operation on the remote $D S A)+$ time required to merge the results and return the result to the $D U A$

$$
T=P_{c}+C_{1}+T_{o 1}+r m_{1}+P_{1}
$$

$P_{c}=$ Total protocol overhead in the client $(D U A)$.

$C_{1}=$ Round trip communication delay between $D U A$ and $D S A_{1}$.

$T_{o 1}=$ Total time required by the $D S A_{1}$ to complete the operation including chaining.

$r m_{1}=$ Time required to merge the results.

$P_{1}=$ Total protocol overhead in the $D S A_{1}$ (including the queuing delay). 
$T_{o 1}$ consists of three parts. Name resolution time in the first $D S A+$ time required to bind to remote $D S A+$ time required to perform the operation on the remote $D S A$.

$$
\begin{aligned}
& T_{o 1}=N_{1}+T_{b}+T_{o} \\
& T_{b}=b f_{1}+C_{12}+P_{2}+b_{2}+P_{1} \\
& T_{o}=q f_{1}+C_{12}+P_{2}+N_{2}+D_{2}+P_{1}
\end{aligned}
$$

$N_{1}=$ time required for name resolution in $D S A_{1}$.

$b f_{1}=$ Time taken to form the bind request.

$C_{12}=$ round trip communication delay between $D S A_{1}$ to $D S A_{2}$.

$P_{2}=$ protocol over head in $D S A_{2}$ (including the queuing delay).

$b_{2}=$ time required to perform bind operation.

$q f_{1}=$ time required to form the query.

$N_{2}=$ time required for name resolution in $D S A_{2}$.

$D_{2}=$ Time required to perform database operation o in $D S A_{2}$.

Thus the total time is given by:

$$
T=P_{c}+C_{1}+3 P_{1}+N_{1}+b f_{1}+2\left(C_{12}+P_{2}\right)+b_{2}+q f_{1}+N_{2}+D_{2}+r m_{1}
$$

Now consider a general case with the chaining goes to an arbitrary depth, say $n$ DSAs. All but the last $D S A$ act as relays to pass the query to the next $D S A$ and all but the first $D S A$ requires binding. It should be noted that the unbind time will not affect the total response time as unbinding is done after sending the result to the $D U A$ or to the previous $D S A$ in the chain. Thus the following actions are repeated in each $D S A$ except the last one:

- resolve the name,

- bind to next $D S A$,

- chain the operation on the next $D S A$ in the chain, and

- merge the result obtained and pass it back to the previous $D S A$.

Thus the total time taken is given by: 


$$
\begin{gathered}
T=P_{c}+C_{1}+3 P_{1}+N_{1}+b f_{1}+2 C_{12}+q f_{1}+\sum_{k=2}^{n}\left(P_{k}+b_{k}\right)+ \\
\sum_{k=2}^{n-1}\left[3 P_{k}+N_{k}+b f_{k}+2 C_{k(k+1)}+r m_{k}\right]+P_{n}+N_{n}+D_{n}
\end{gathered}
$$

\subsection{Case III - Referral}

In this case the $D S A$ returns a referral to the $D U A$, which in turn uses this referral to contact another $D S A$ which can answer the query. The interactions that are taking place are given in Figure 4.

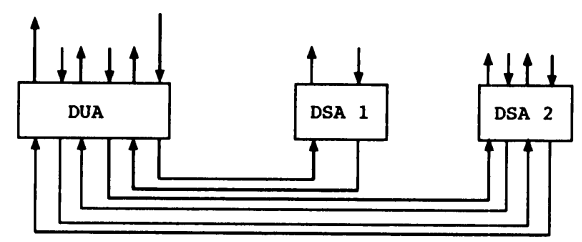

Figure 4: Multiple DSAs, Referral mode of interaction

The operation can be split into the following sub-operations:

- The $D U A$ sends the request to $D S A_{1}$ which returns a referral.

- $D U A$ uses these referral and binds to a $D S A$ whose address is given in the referral.

- $D U A$ sends the operation request to the second $D S A$ which services it and returns the result.

Timing for each of these operations are given below.

$T_{1}=P_{c}+C_{1}+P_{1}+N_{1}$

$T_{2 b}=b f_{c}+P_{c}+C_{2}+P_{2}+b_{2}$

$T_{2 o}=q f_{c}+P_{c}+C_{2}+P_{2}+N_{2}+D_{2}$

Thus the total time $\mathrm{T}$ is given by:

$T=3 P_{c}+C_{1}+P_{1}+N_{1}+q f_{c}+b f_{c}+2\left(C_{2}+P_{2}\right)+b_{2}+N_{2}+D_{2}$

If the referral extends to $n D S A s$, the total time required is given by: 


$$
\begin{gathered}
T=(2 n-1) P_{c}+(n-1) b f_{c}+n * q f_{c}+\sum_{k=2}^{n}\left(C_{k}+P_{k}+b_{k}\right)+ \\
\sum_{k=1}^{n}\left(C_{k}+P_{k}+N_{k}\right)+D_{n}
\end{gathered}
$$

Hitherto, we developed a deterministic model to predict $T$, the time required to perform the directory operations, in terms of the parameters $P_{c}, P_{1}, P_{2}, N_{1}, N_{2}, C_{1}, C_{2}, C_{12}$, $D_{1}, D_{2}, b_{1}, b f_{c}, b f_{1}, q f_{c}, q f_{1}$ and $r m_{1}$. The completion of the model requires estimation of these parameters.

\section{PARAMETER ESTIMATION OF THE MODEL}

A series of experiments were designed to measure the parameters identified in the section 3. Routines for logging the time are included at various points in both $D U A$ and $D S A$. A set of ten experiments are conducted and the average values are taken. The parameters required by the model is estimated from these measured values. Figure 5 shows the architecture of $D S A$ and $D U A$ in the experimental setup. The shaded area indicates the routines added to measure the timings.

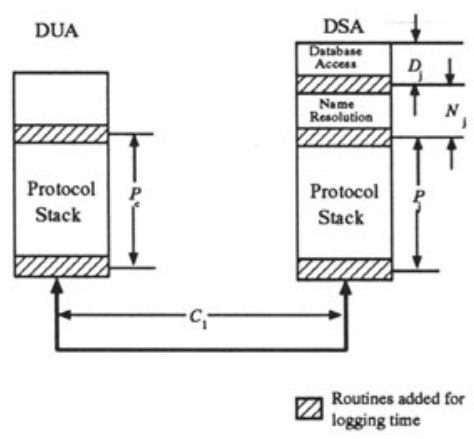

Figure 5: Experimental Setup

In the $D U A$, the time is logged in just before the query is passed onto the protocol stack and once again before the packet is passed to the network. Similarly, in the DSA, time is logged when a packet is received from the network, before and after the name resolution and during the database access. That way, database access time and the name resolution time can be calculated. As the clocks in the two systems are not synchronized with each other, it is not possible to measure the oneway communication delay. But by measuring the packet transmission time and packet reception time at both ends, it is possible to measure the round trip communication time. 


\begin{tabular}{|c|c|c|c|c|c|}
\hline $\begin{array}{c}\text { Experiment } \\
\text { Number }\end{array}$ & $D S A_{1}$ & $D S A_{2}$ & $D U A$ & $\begin{array}{c}\text { Where } \\
\text { reported }\end{array}$ & Mode \\
\hline 1 & IITM & N/A & IITM & Table 2 & N/A \\
2 & UMD & N/A & IITM & Table 3 & N/A \\
3 & IITM & UMD & IITM & Table 4 & Chaining \\
4 & IITM & UMD & IITM & Table 5 & Referral \\
\hline
\end{tabular}

Table 1: Experiments Conducted.

The DIT structure used for the measurements is shown in the Figure 6. Experiments were conducted with different network topologies and different modes of interactions. The experiments conducted are listed in the Table 1.

The database used for the experiments is generated from a subscriber database used by telecommunication department. The entries in the database are duplicated to create bigger databases. An unique identifier is appended so as to make the name of every subscriber unique. This name is then used as the RDN of the entry. Subordinates of a given entry is stored in a separate file and this file is indexed using the B-Tree++ algorithm, with RDN as the key.

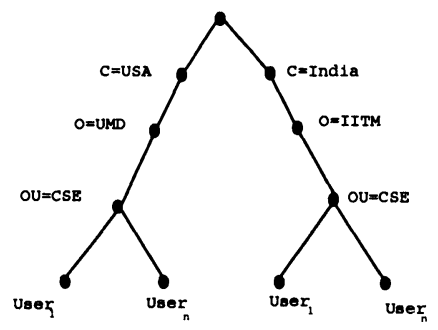

Figure 6: DIT structure

Tables 2 and 3 present the measurements that are taken between three different machines in the internet. Table 2 gives the measurements with $D S A$ running in the Sparc machine in IIT, Madras, India and DUA running in the same LAN in a Tektronix machine in IIT, Madras, India.

Table 3 shows the measurements with the $D S A$ running in the Ultrix machine in University of Maryland, USA, and the DUA running in the Sparc machine in IIT, Madras, India. All values given in the tables are the average over a set of ten measurements.

Table 4 shows the measurements when the directory is distributed among two systems in the internet, using chaining mode of interaction between the DSAs. The DSAs were running in the Ultrix machine in University of Maryland, USA and in the Sparc machine 
in IIT, Madras and DUA in the tektronix machine in IIT, Madras. Table 5 shows the measurements done with the same configuration using the referral mode of interaction.

From the tables, the following observations can be made:

- The time required to perform the database operation depends on the type of operation. From the tables, it can be seen that the most time consuming directory operation is modifyrdn operation. This is because the modifyrdn operation requires searching the database twice to perform the operation - once to check the existance of the entry whose modification is requested and then to confirm that no other entry with the same name exists.

- The database operation time and the name resolution time increases with the increase in the number of entries in the directory database. This is because of the increase in the depth of the B-Tree.

- The time required to perform the list operation does not depends on the database size. As the number of subordinates of the base object is always larger than the sizelimit parameter, the list operation always returns the first few entries and returns the sizelimit exceeded error. This is a limitation of the 1988 standard of X.500[CCITT (1988)], which has been overcome in 1993 version[CCITT (1993)].

The estimated values of model parameters are used in predicting the directory operation time $T$. In all the experiments, the directory operation time $T$ was also measured. Table 6 gives the parameters related to directory operations. The values $D_{1}$ is the database operation time and $N_{1}$ the name resolution time in the $D S A$ where the entry is located. Table 7 gives the $D S A-D U A$ interaction parameters. The values presented include the bind query formation time, the bind operation time, the query formation time and the result merging time. Table 8 gives the parameters for communication delay and protocol overhead. In the table, $C_{l}$ is the communication delay in a LAN environment and $C_{i}$ is the communication delay in an internet environment.

The variations in the predicted value and the actually measured value are computed. The error rate is calculated as (predicted value - measured value) / predicted value * 100. The error rate is plotted against database size for all directory operation for all the four experiments in the Figure 8, 9, 10, and 11. The graph given in the Figure 9 shows the variation in the percentage error rate with the number of entries for experiment 2, with no referral or chaining in an internet environment. From this graph, it is evident that the percentage error rate decreases as the number of entries increase. The estimated database access time is more accurate than the round trip communication time. The round trip communication time is considerably smaller when compared to the database access time, when the database size reaches 50,000 . Thus the percentage variation in the predicted response time will become smaller with the increase in the number of entries 
in the directory. This can be predicted accurately. But the round trip communication time across the internet cannot be predicted accurately [Sanghi et al.(1993)]. When the database size is small the round trip communication time will be small and thus the percentage variation in the predicted value from the measured value will be high.

Figure 10 shows the percentage variation of error with increase in database size for chaining mode of interaction. The variation in this case is also small for large database size. In our experiments, the $D U A$ and one $D S A$ was in the same LAN. Thus the communication delay between the $D U A$ and the first $D S A$ will be very less. Figure 11 in the appendix gives the percentage variations of the error with increase in database size for referral mode of interaction. Again, the variation in the predicted value from the actual measurements decreases with increase in the database size.

\section{APPLICATION OF THE MODEL FOR ESTIMATING THE NAME LOOKUP TIME}

We now use the deterministic model to predict the expected response time for read operation in a national network. Two different DIT structures are considered and the response time are computed for each structure with the help of the model.

We consider the $E R N E T^{4}$ topology, and estimate the response time using the analytical model discussed in the section 3.

The network topology of ERNET is shown in the Figure 7a. ERNET consists of 8 different sites, connected by point-to-point links of capacity $9.6 \mathrm{kbps}$. We estimate the average response time for a query given to any one of the site in the ERNET to access the data in any other site. The label on the arcs connecting the sites are the round trip time between the nodes. The actual communication delay between the sites is measured using the ping command.

Figure $7 \mathrm{~b}$ shows the logical tree structure used to estimate the response time for a directory operation. The average response time estimated using the analytical model for the ERNET directory for the DIT structure shown in the Figure 7b is shown in the Table 9. Figure 7c shows another logical directory tree structure with the first level $D S A$ located at a different site. The response time with this DIT configuration is given in the Table 10. The values given are for a single lookup (read) operation with 500 entries in each $D S A$. The value in column $\mathrm{i}$ and row $\mathrm{j}$ indicates the time required to access an object in site $\mathrm{i}$ by a $D U A$ in site j. We assume that $D S A s$ stores no cross references to any other $D S A s$, thus the name resolution is done by strict tree traversal.

The Table 9 and 10 show the response time for directory operations with different DIT structures. The readings given are the estimated time for directory read operations. The difference in the readings clearly show the advantage of selecting a proper DIT structure

\footnotetext{
${ }^{4}$ Education and Research NETwork, setup by Department of Electronics and UNDP.
} 


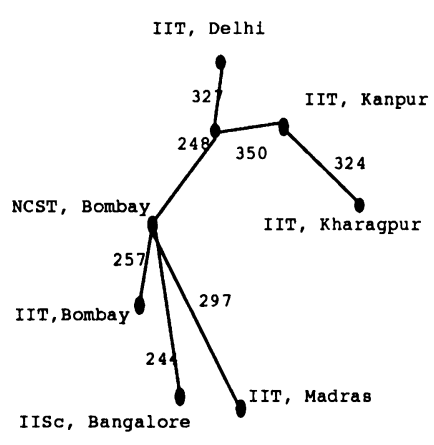

a. ERNET Topology

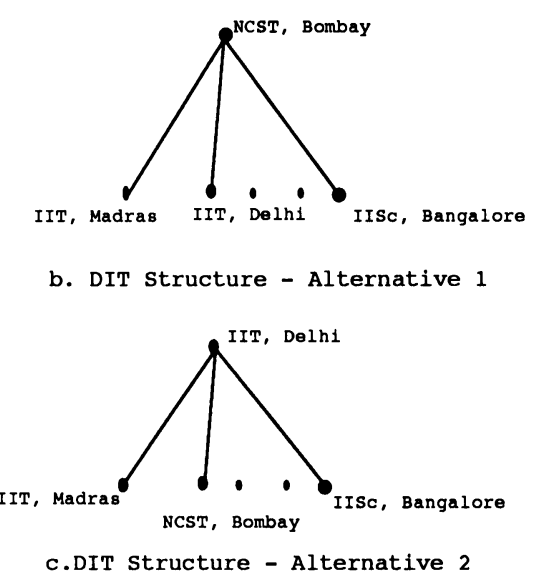

Figure 7: ERNET directory

for a given topology. The directory structure given in Figure $7 \mathrm{~b}$ is best among the two topologies as it gives the minimum response time.

\section{CONCLUSION}

In this paper, we presented a simple deterministic model for predicting the performance of the X.500 directory. This model will be helpful for the X.500 directory managers to tune the directory to get a better performance. The model assists the directory manager in estimating the critical database size and the optimal topology to be used. The model is validated by comparing the results predicted using the model and the actual measurements carried out in the internet with a large database. The percentage deviation in the predicted results from the measured results was found to be very less thereby confirming the usefulness of the model. Also, the deviation of the predicted results from the measured value decreases with increase in the database size because the variations in the communication delay, which is an unpredictable factor, will be a small percentage of the total time required to perform the directory operation. The model is used to select the optimal topology for DIT, when the physical interconnection topology of a large national network is given.

\section{References}

[CCITT (1988)] CCITT. Data Communication Networks, Message Handling Systems, Recommendations X.400 - X.420. 1988. 
[CCITT (1988)] CCITT. The Directory - Overview of Concepts, Models and Services, Recommendations X.500 - X.521. 1988.

[CCITT (1993)] CCITT. The Directory - Overview of Concepts, Models and Services, Draft Recommendations X.500 - X.521. 1993.

[Goodman (1992)] David Goodman. PARADISE International Report 4. PARADISE Pilot Group, University College London, November 1992.

[Sanghi et al.(1993)] Dheraj Sanghi, Olafur Gudmundsson, Ashok Agarwala. Study of network dynamics. Computer Networks and ISDN Systems, 26:371-378, 1993.

[Comer (1979)] Douglas Comer. The Ubiquitous B-trees. ACM Computing Surveys, 11(2):121-137, June 1979.

[Huitema (1989)] G. Huitema. The X.500 Directory Services. Computer Networks and ISDN Systems, 16:161-166, September 1989.

[Neufeld (1992)] Gerald Neufeld, Barry Brachman, Murray Goldberg, Duncan Stickings. The EAN X.500 Directory Services. Internetworking : Research and experience, 3:55-81, 92.

[Mansfield et al.(1993)] Glenn Mansfield, K Jayanthi, K Higuchi, Thomas Johannsen, Y Nemoto. Mapping communication networks in the directory. Computer networks and ISDN Systems, 26:327-335, 1993.

[Hong et al.(1992a)] James W Hong, Micheal A Bauer, J Michael Bennet. Integration of Directory Services in the network management framework. In Third International Symposium on Integrated Network Management, April 1992.

[Hong et al.(1992b)] James W Hong, Micheal A Bauer, J Micheal Bennet. Integragion of the Directory Services in the Distributed systems management. In International Conference on Parallel and Distributed Systems, 1992.

[Barker (1992)] Paul Barker. DIT Structures to support searching for Bibilographic information and information resources. Technical report, University College London, December 1992.

[Barker (1993)] Paul Barker. Implementing FTP archive searching using X.500. Internet Draft : OSI-DS 40, March 1993.

[Bumbulis et al.(1993)] P.J. Bumbulis, D.D. Cowan, C.M. Durance, T.M. Stepien. An Introduction to the OSI Directory Services. Computer Networks and ISDN Systems, 26:239-249, 1993. 
[Sriram (1991)] R Sriram. Studies in OSInet Directory Services. Master's thesis, Indian Institute of Technology Madras, January 1991.

[Sriram et al.(1994)] R Sriram, S V Raghavan. Example implementation of Name Services using Directory Services. To appear in Computer Networks and ISDN Systems, 1994.

[Raghavan (1990)] S.V. Raghavan. Solutions to Local Area Networks, The Indian Context. Tata McGraw Hill Publishing Company Limited, 1990.

[Rennesse et al.] Robbert Van Rennesse, Andrew S Tanenbaum. A Directory service supporting avaliability and consistency. Vrije Universiteit, Amsterdam.

[Raghavan et al.(1992)] S. V. Raghavan, R. Sriram. Implementation of X.500 Directory Services in OSInet. IETE Technical Review, 9(3), May - June 1992.

[Raghavan (1993a)] S.V. Raghavan. OSInet: Open Systems Interconnection network Software:: Description of its Protocol Architecture, Design, Implementation, Application and Performance. Technical Report ERNET/06/93/IITM, CSE/NSL/08/93/IITM, Indian Institute of Technology, Madras, April 1993.

[Raghavan et al.(1993b)] S.V. Raghavan, B. Prabhakaran, K. Shivkumar. An enhanced B tree design for databases. Technical Report ERNET/10/93/IITM CSE/NSL/12/93/IITM, Indian Institute of Technology, Madras, May 1993.

\section{AUTHOR INFORMATION}

Rajeev P.V. obtained his Bachelor's degree in 1991 from the University of Calicut, India in the field of Computer Science and Engineering. He completed his Master's in Computer Science and Engineering from Indian Institute of Technology, Madras, India. He was working as a research fellow in Networks Systems Laboratory, Department of Computer Science, Indian Institute of Technology, Madras, India. At present, he is with Hughes Software Systems, Delhi. His research interests are networks and multimedia systems.

S.V. Raghavan is on the faculty of the Department of Computer Science and Engineering, Indian Institute of Technology, Madras. He is also the Chief Investigator of the project on Education and Research in Computer Networking jointly sponsored by the Department of Electronics, Government of India and the United Nations Development Programme. He is a life member of the Computer Society of India, a member of the Institution of Engineers and a fellow of institute of Electronics and Telecommunication Engineers. He is presently serving on the Board of Editors of the journal of IETE for 
computers and control. He is also a member of the Editorial Advisory Board for Computer Communications, Butterwoth-Heinemann Ltd. His research interests are networks, protocols, multimedia systems and performance.

Ashok K. Agarwala is a Professor of Computer Science at the University of Maryland, College Park. For the past twenty years he has been actively involved in the research in various aspects of computer system design, implementation and performance. He received his the B.Sc. degree from Agra University in 1960, the B.E degree in Electrical Technology, and the M.E. degree in Applied Electronics and Servomechanisms from Indian Institute of Science in 1963 and 1965, respectively. He earned the A.M. and Ph.D. degrees in Applied Mathematics from Harward University, Cambridge, MA in 1970. Dr. Agrawala is fellow of the IEEE, and a member of the Association for Computing Machinery, American Association for the Advancement of Science Xi. 


\begin{tabular}{|c|c|c|c|c|c|c|c|}
\hline \multicolumn{8}{|c|}{$\begin{array}{c}\text { DSA running in Sparc, IIT Madras and DUA in Tektronix, IIT Madras } \\
\text { Number of Entries }=5000\end{array}$} \\
\hline Time & Read & Compare & List & Add & Modify & Remove & ModifyRDN \\
\hline$D_{1}$ & 531 & 337 & 301 & 1427 & 1011 & 210 & 2203 \\
\hline$N_{1}$ & 1096 & 1118 & 781 & 1219 & 1137 & 1060 & 1256 \\
\hline$P_{1}$ & 33 & 31 & 20 & 31 & 19 & 29 & 26 \\
\hline$C_{1}$ & 38 & 29 & 27 & 38 & 25 & 31 & 38 \\
\hline$P_{c}$ & 18 & 19 & 17 & 29 & 17 & 22 & 17 \\
\hline $\mathbf{T}$ & 1710 & 1534 & 1146 & 2744 & 2209 & 1352 & 3540 \\
\hline \multicolumn{8}{|c|}{ Number of Entries $=10000$} \\
\hline$D_{1}$ & 593 & 392 & 1610 & 1213 & 232 & 1932 & 1486 \\
\hline$N_{1}$ & 1461 & 1460 & 798 & 1432 & 1472 & 1503 & 1496 \\
\hline$P_{1}$ & 34 & 25 & 25 & 22 & 30 & 25 & 27 \\
\hline$C_{1}$ & 38 & 38 & 37 & 32 & 26 & 33 & 29 \\
\hline$P_{c}$ & 25 & 25 & 18 & 18 & 21 & 17 & 27 \\
\hline$i^{c}$ & 2151 & 1940 & 1250 & 3114 & 2762 & 1810 & 3511 \\
\hline \multicolumn{8}{|c|}{ Number of Entries $=25000$} \\
\hline$D_{1}$ & 381 & 410 & 363 & 3793 & 2478 & 241 & 3976 \\
\hline$N_{1}$ & 2262 & 2145 & 728 & 2453 & 2320 & 2413 & 2417 \\
\hline$P_{1}$ & 23 & 32 & 32 & 34 & 34 & 31 & 33 \\
\hline$C_{1}$ & 26 & 35 & 37 & 38 & 31 & 36 & 29 \\
\hline$P_{c}$ & 19 & 30 & 20 & 20 & 30 & 18 & 25 \\
\hline 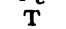 & 2911 & 2652 & 1201 & 6338 & 4893 & 2739 & 6479 \\
\hline \multicolumn{8}{|c|}{ Number of Entries $=80000$} \\
\hline & 512 & 409 & 410 & 6781 & 4823 & 303 & 7013 \\
\hline$N_{1}$ & 3813 & 3623 & 813 & 3713 & 3691 & 3686 & 3594 \\
\hline$P_{1}$ & 29 & 25 & 32 & 19 & 34 & 34 & 24 \\
\hline$C_{1}$ & 26 & 30 & 35 & 27 & 33 & 34 & 26 \\
\hline$P_{c}$ & 22 & 18 & 30 & 18 & 25 & 26 & 22 \\
\hline & 4402 & 4105 & 1320 & 10558 & 8706 & 4083 & 10679 \\
\hline
\end{tabular}

All values in milliseconds

Table 2: DSA and DUA on LAN.

\begin{tabular}{|c|c|c|c|c|c|c|c|}
\hline \multicolumn{8}{|c|}{$\begin{array}{l}\text { DUA running in Sparc, IIT, Madras and DSA in Univ. of Maryland } \\
\text { Number of Entries }=5000\end{array}$} \\
\hline Time & Read & Compare & List & Add & Modify & Remove & ModifyRDN \\
\hline$D_{1}$ & 531 & 320 & 343 & 1461 & 1078 & 180 & 2304 \\
\hline$N_{1}$ & 1220 & 1180 & 712 & 1207 & 1230 & 1141 & 1162 \\
\hline$P_{1}$ & 26 & 29 & 27 & 31 & 27 & 26 & 29 \\
\hline$C_{1}$ & 1290 & 1214 & 1195 & 1175 & 1108 & 1178 & 1187 \\
\hline$P_{c}$ & 16 & 21 & 18 & 29 & 22 & 23 & 23 \\
\hline $\mathbf{T}$ & 3126 & 2764 & 2293 & 3903 & 3262 & 2558 & 4695 \\
\hline \multicolumn{8}{|c|}{ Number of Entries $=10000$} \\
\hline$\overline{D_{1}}$ & 532 & 393 & 327 & 1772 & 1246 & 210 & 2802 \\
\hline$N_{1}$ & 1400 & 1364 & 719 & 1767 & 1879 & 1364 & 1782 \\
\hline$P_{1}$ & 24 & 27 & 23 & 31 & 29 & 20 & 32 \\
\hline$C_{1}$ & 1239 & 1206 & 1195 & 1223 & 1156 & 1259 & 1150 \\
\hline$P_{c}$ & 23 & 20 & 27 & 22 & 18 & 23 & 21 \\
\hline & 3318 & 3010 & 2270 & 5015 & 4628 & 2886 & 5067 \\
\hline \multicolumn{8}{|c|}{ Number of Entries $=25000$} \\
\hline$D_{1}$ & 546 & 403 & 308 & 3632 & 2590 & 230 & 3816 \\
\hline$N_{1}$ & 2561 & 2232 & 738 & 2762 & 2260 & 2123 & 2974 \\
\hline$P_{1}$ & 27 & 23 & 29 & 33 & 29 & 23 & 29 \\
\hline$C_{1}$ & 1319 & 1294 & 1299 & 1226 & 1213 & 1302 & 1264 \\
\hline $\boldsymbol{P}_{c}$ & 21 & 18 & 21 & 29 & 27 & 19 & 23 \\
\hline$\pi$ & 4464 & 3952 & 2395 & 7682 & 6122 & 3897 & 7805 \\
\hline \multicolumn{8}{|c|}{ Number of Entries $=\mathbf{0 0 0 0 0}$} \\
\hline$D_{1}$ & 573 & 420 & 380 & 6585 & 4833 & 287 & 6952 \\
\hline$N_{1}$ & 3905 & 3685 & 790 & 3917 & 3729 & 3519 & 3923 \\
\hline$P_{1}$ & 29 & 23 & 31 & 28 & 26 & 29 & 28 \\
\hline$C_{1}$ & 1278 & 1123 & 1145 & 1292 & 1240 & 1153 & 1231 \\
\hline$P_{c}$ & 21 & 18 & 27 & 21 & 23 & 22 & 19 \\
\hline & 5808 & 5226 & 2363 & 11841 & 9851 & 5010 & 12150 \\
\hline
\end{tabular}

Table 3: DSA and DUA Across Internet. 


\begin{tabular}{|c|c|c|c|c|c|c|c|}
\hline \multicolumn{8}{|c|}{$\begin{array}{c}\text { DSAs in Maryland and Sparc, IIT, Madras and DUA in Tektronix } \\
\text { Chaining mode of interaction } \\
\text { Number of Entries }=5000\end{array}$} \\
\hline Time & Read & Compare & List & Add & Modify & Remove & ModifyRDN \\
\hline$D_{2}$ & 618 & 526 & 428 & 1198 & 1063 & 230 & 1839 \\
\hline$N_{2}$ & 1003 & 1029 & 721 & 1102 & 1121 & 1017 & 1482 \\
\hline$N_{1}$ & 307 & 314 & 418 & 472 & 381 & 238 & 325 \\
\hline$P_{1}$ & 26 & 22 & 29 & 30 & 22 & 28 & 24 \\
\hline$P_{2}$ & 20 & 21 & 28 & 21 & 30 & 20 & 24 \\
\hline$P_{\mathrm{c}}$ & 19 & 23 & 19 & 20 & 23 & 22 & 20 \\
\hline$C_{1}$ & 34 & 34 & 36 & 26 & 29 & 28 & 31 \\
\hline$C_{12}$ & 1162 & 1227 & 1241 & 1181 & 1286 & 1280 & 1143 \\
\hline$b f_{1}$ & 38 & 32 & 39 & 30 & 31 & 34 & 35 \\
\hline$q f_{1}$ & 45 & 38 & 35 & 42 & 39 & 45 & 48 \\
\hline$r m_{1}$ & 80 & 36 & 49 & 46 & 38 & 39 & 30 \\
\hline$b_{2}$ & 122 & 110 & 112 & 142 & 124 & 106 & 146 \\
\hline$\overline{\mathbf{T}}$ & 4678 & 4704 & 4482 & 5572 & 5547 & 4443 & 6362 \\
\hline \multicolumn{8}{|c|}{ Number of Entries $=10000$} \\
\hline$\overline{D_{2}}$ & 597 & 387 & 441 & 1780 & 1312 & 348 & 1899 \\
\hline$N_{2}$ & 1492 & 1480 & 892 & 1471 & 1398 & 1801 & 1496 \\
\hline$N_{1}$ & 318 & 329 & 393 & 403 & 307 & 321 & 481 \\
\hline$P_{1}$ & 28 & 29 & 30 & 20 & 33 & 20 & 34 \\
\hline $\boldsymbol{P}_{\mathbf{2}}$ & 30 & 33 & 26 & 27 & 21 & 33 & 22 \\
\hline$P_{c}$ & 22 & 18 & 24 & 23 & 20 & 18 & 18 \\
\hline$C_{1}$ & 32 & 35 & 35 & 25 & 32 & 31 & 30 \\
\hline$C_{12}$ & 1286 & 1156 & 1108 & 1259 & 1257 & 1295 & 1291 \\
\hline$b f_{1}$ & 33 & 39 & 33 & 33 & 35 & 30 & 35 \\
\hline$q f_{1}$ & 45 & 49 & 35 & 48 & 49 & 44 & 42 \\
\hline$r m_{1}$ & 48 & 38 & 54 & 49 & 35 & 43 & 36 \\
\hline$b_{2}$ & 117 & 107 & 143 & 100 & 120 & 114 & 125 \\
\hline$\overline{\mathbf{T}}$ & $\mathbf{5 4 2 0}$ & 4947 & 4408 & 6564 & 5963 & 5166 & 6890 \\
\hline \multicolumn{8}{|c|}{ Number of Entries $=26000$} \\
\hline$D_{2}$ & 588 & 476 & 480 & 3578 & 2489 & 480 & 3857 \\
\hline $\mathrm{N}_{2}$ & 2378 & 2247 & 760 & 2782 & 2490 & 2389 & 2415 \\
\hline$N_{1}$ & 321 & 370 & 280 & 420 & 338 & 264 & 407 \\
\hline$P_{1}$ & 33 & 32 & 31 & 32 & 22 & 31 & 33 \\
\hline $\boldsymbol{P}_{2}$ & 23 & 32 & 27 & 23 & 28 & 27 & 27 \\
\hline $\boldsymbol{P}_{c}$ & 18 & 19 & 24 & 20 & 19 & 24 & 18 \\
\hline$C_{1}$ & 25 & 38 & 37 & 34 & 36 & 37 & 30 \\
\hline$C_{12}$ & 1304 & 1212 & 1162 & 1257 & 1222 & 1162 & 1264 \\
\hline$b f_{1}$ & 38 & 30 & 37 & 39 & 34 & 37 & 33 \\
\hline$q f_{1}$ & 37 & 39 & 47 & 36 & 43 & 47 & 38 \\
\hline$r m_{1}$ & $\mathbf{5 3}$ & 52 & 51 & 32 & 36 & 51 & 33 \\
\hline$b_{2}$ & 115 & 122 & 106 & 107 & 114 & 106 & 133 \\
\hline$T$ & 6326 & 5977 & 4293 & 9704 & 8165 & 5906 & 9645 \\
\hline \multicolumn{8}{|c|}{ Number of Entries $=80000$} \\
\hline$D_{2}$ & 621 & 508 & 405 & 6817 & 4293 & 421 & 6983 \\
\hline$N_{2}$ & 3873 & 3692 & 821 & 3985 & 3961 & 3796 & 3975 \\
\hline$N_{1}$ & 298 & 264 & 269 & 347 & 469 & 256 & 442 \\
\hline$P_{1}$ & 20 & 28 & 31 & 32 & 22 & 34 & 22 \\
\hline $\boldsymbol{P}_{\mathbf{2}}$ & 26 & 33 & 20 & 31 & 33 & 24 & 26 \\
\hline $\boldsymbol{P}_{\mathrm{c}}$ & 21 & 22 & 24 & 23 & 21 & 19 & 21 \\
\hline$C_{1}$ & 30 & 36 & 27 & 26 & 38 & 27 & 26 \\
\hline$C_{12}$ & 1142 & 1198 & 1313 & 1235 & 1323 & 1243 & 1248 \\
\hline$b f_{1}$ & 37 & 30 & 31 & 30 & 37 & 30 & 31 \\
\hline$q f_{1}$ & 38 & 35 & 46 & 41 & 35 & 48 & 36 \\
\hline$r m_{1}$ & 60 & 40 & 44 & 40 & 48 & 35 & 52 \\
\hline$b_{2}$ & 138 & 135 & 147 & 124 & 117 & 108 & 128 \\
\hline$T$ & 7502 & 7308 & 4573 & 14061 & 11797 & 7376 & 14308 \\
\hline
\end{tabular}

All values in milliseconds

Table 4: DSAs Across Internet with Chaining + DUA. 


\begin{tabular}{|c|c|c|c|c|c|c|c|}
\hline \multicolumn{8}{|c|}{$\begin{array}{c}\text { DSAs in Maryland and Sparc, ITT, Madras and DUA in Tektronix } \\
\text { Referral mode of interaction } \\
\text { Number of Entries }=5000\end{array}$} \\
\hline Time & Read & Compare & Cist & Add & Modify & Remove & ModifyRDN \\
\hline$D_{2}$ & 639 & 570 & 400 & 1247 & 1036 & 242 & 1793 \\
\hline $\mathrm{N}_{2}$ & 1027 & 1001 & 658 & 1051 & 1150 & 1073 & 1407 \\
\hline$N_{1}$ & 329 & 360 & 394 & 463 & 414 & 217 & 334 \\
\hline$P_{1}$ & 25 & 34 & 32 & 34 & 26 & 23 & 23 \\
\hline$P_{2}$ & 34 & 27 & 33 & 33 & 23 & 21 & 23 \\
\hline$P_{c}$ & 20 & 22 & 18 & 22 & 24 & 23 & 22 \\
\hline$C_{1}$ & 38 & 33 & 25 & 37 & 30 & 33 & 30 \\
\hline$C_{2}$ & 1228 & 1222 & 1233 & 1154 & 1136 & 1164 & 1184 \\
\hline$b f_{c}$ & 32 & 38 & 34 & 32 & 39 & 39 & 38 \\
\hline$q f_{c}$ & 43 & 36 & 41 & 48 & 46 & 40 & 38 \\
\hline$b_{2}$ & 115 & 117 & 138 & 116 & 138 & 138 & 112 \\
\hline $\mathbf{T}$ & 4832 & 4753 & 4308 & 5468 & 5269 & 4244 & 6275 \\
\hline \multicolumn{8}{|c|}{ Number of Entries $=10000$} \\
\hline$D_{2}$ & 607 & 384 & 448 & 1785 & 1296 & 299 & 1850 \\
\hline$N_{2}$ & 1430 & 1512 & 918 & 1445 & 1391 & 1483 & 1510 \\
\hline$N_{1}$ & 284 & 369 & 396 & 432 & 349 & 349 & 496 \\
\hline$P_{1}$ & 25 & 32 & 31 & 22 & 33 & 21 & 24 \\
\hline$P_{2}$ & 32 & 33 & 29 & 29 & 24 & 29 & 30 \\
\hline$P_{c}$ & 22 & 23 & 21 & 23 & 24 & 23 & 18 \\
\hline$C_{1}$ & 39 & 37 & 26 & 25 & 38 & 28 & 31 \\
\hline$C_{2}$ & 1223 & 1281 & 1154 & 1149 & 1167 & 1120 & 1130 \\
\hline$b f_{c}$ & 33 & 32 & 38 & 36 & 36 & 32 & 32 \\
\hline$q f_{c}$ & 38 & 41 & 49 & 47 & 38 & 35 & 44 \\
\hline$b_{2}$ & 100 & 133 & 135 & 141 & 134 & 139 & 104 \\
\hline 2 & 6132 & $\mathbf{5 2 3 7}$ & 4470 & 6358 & 6769 & 4753 & 6465 \\
\hline \multicolumn{8}{|c|}{ Number of Entries $=25000$} \\
\hline$D_{2}$ & 891 & 449 & 444 & 3633 & 2486 & 494 & 3836 \\
\hline$N_{2}$ & 2336 & 2211 & 726 & 2711 & 2465 & 2348 & 2417 \\
\hline$N_{1}$ & 303 & 407 & 302 & 383 & 332 & 222 & 372 \\
\hline$P_{1}$ & 25 & 34 & 21 & 25 & 23 & 21 & 32 \\
\hline$P_{2}$ & 23 & 23 & 26 & 24 & 26 & 34 & 26 \\
\hline$P_{c}$ & 20 & 19 & 19 & 24 & 20 & 23 & 22 \\
\hline$C_{1}$ & 25 & 26 & 33 & 25 & 35 & 25 & 37 \\
\hline$C_{2}$ & 1114 & 1343 & 1100 & 1165 & 1157 & 1211 & 1134 \\
\hline$b f_{c}$ & 35 & 36 & 34 & 37 & 33 & 36 & 37 \\
\hline$q f_{c}$ & 48 & 38 & 40 & 44 & 42 & 39 & 35 \\
\hline$b_{2}$ & 128 & 114 & 145 & 113 & 111 & 117 & 120 \\
\hline & 6825 & 6104 & 4054 & 9321 & 7953 & 5861 & 9272 \\
\hline \multicolumn{8}{|c|}{ Number of Entries $=\mathbf{5 0 0 0 0}$} \\
\hline$D_{2}$ & 670 & 499 & 386 & 6816 & 4266 & $\mathbf{4 3 2}$ & 6960 \\
\hline$N_{2}$ & 3918 & 3624 & 760 & 4025 & 3920 & $\mathbf{3 7 3 7}$ & 3998 \\
\hline$N_{1}$ & 264 & 302 & 244 & 361 & 483 & 271 & 461 \\
\hline$P_{1}$ & 21 & 23 & 30 & 32 & 30 & 27 & 25 \\
\hline$P_{2}$ & 23 & 30 & 32 & 25 & 29 & 33 & 30 \\
\hline$P_{c}$ & 22 & 21 & 23 & 24 & 18 & 19 & 24 \\
\hline$C_{1}$ & 26 & 31 & 29 & 39 & 31 & 30 & 27 \\
\hline$C_{2}$ & 1285 & 1114 & 1282 & 1240 & 1289 & 1127 & 1243 \\
\hline$b f_{c}$ & 31 & 30 & 34 & 35 & 38 & 38 & 39 \\
\hline$q f_{c}$ & 37 & 46 & 47 & 39 & 42 & 35 & 45 \\
\hline$b_{2}$ & 104 & 116 & 146 & 145 & 104 & 116 & 116 \\
\hline 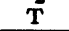 & 7753 & 7022 & 4373 & 14094 & 11603 & 7063 & 14289 \\
\hline
\end{tabular}

All values in milliseconds

Table 5: DSAs Across Internet with Referral + DUA. 


\begin{tabular}{|c|c|c|c|c|c|c|}
\hline $\begin{array}{c}\text { Variable } \\
\text { Estimated }\end{array}$ & Read & Compare & Add & Modify & Remove & ModifyRDN \\
\hline \multicolumn{7}{|c|}{ Number of entries $=5000$} \\
\hline$D_{1}$ & 531 & 320 & 1500 & 1075 & 200 & 2300 \\
$N_{1}$ & 1220 & 1180 & 1200 & 1230 & 1140 & 1160 \\
\hline \multicolumn{7}{|c|}{ Number of entries $=10,000$} \\
\hline$D_{1}$ & 532 & 393 & 1772 & 1246 & 210 & 2802 \\
$N_{1}$ & 1400 & 1364 & 1767 & 1879 & 1364 & 1782 \\
\hline \multicolumn{7}{|c|}{ Number of entries = 25,000 } \\
\hline$D_{1}$ & 546 & 403 & 3632 & 2590 & 230 & 3815 \\
$N_{1}$ & 2561 & 2232 & 2762 & 2260 & 2123 & 2874 \\
\hline \multicolumn{7}{|c|}{ Number of entries = 50,000 } \\
\hline$D_{1}$ & 673 & 420 & 6583 & 4833 & 287 & 6982 \\
$N_{1}$ & 3905 & 3685 & 3917 & 3729 & 3519 & 3923 \\
\hline
\end{tabular}

Table 6: Estimated Values of Model Parameters Related to Directory Operations.

\begin{tabular}{|c|c|}
\hline Estimated Variable & Value \\
\hline Time to search knowledge information $\left(N_{1}\right)$ & 550 \\
Bind request formation time $\left(b f_{1}\right)$ & 40 \\
Bind time $\left(b_{i}\right)$ & 130 \\
Query Formation time $\left(q f_{1}\right)$ & 45 \\
Result merging time $\left(r m_{1}\right)$ & 45 \\
\hline
\end{tabular}

Table 7: Estimated Values of $D S A$ - DUA interaction parameters.

\begin{tabular}{|c|c|c|c|c|}
\hline $\begin{array}{c}\text { Estimated } \\
\text { Variable }\end{array}$ & $\begin{array}{c}\text { Minimum } \\
\text { Value }\end{array}$ & $\begin{array}{c}\text { Maximum } \\
\text { Value }\end{array}$ & Mean & $\begin{array}{c}\text { Standard } \\
\text { Deviation }\end{array}$ \\
\hline$P_{1}$ & 19 & 34 & 27 & 0.427 \\
$P_{2}$ & 20 & 34 & 26 & 0.588 \\
$P_{c}$ & 16 & 30 & 21 & 0.380 \\
$C_{l}$ & 25 & 39 & 30 & 0.860 \\
$C_{i}$ & 1100 & 1343 & 1198 & 7.020 \\
\hline
\end{tabular}

Table 8: Estimated Values of Protocol Overhead and Communication Delay Parameters.

\begin{tabular}{|c|c|c|c|c|c|c|c|c|}
\hline & ncst & iitm & iitb & II8c & doe & iitkgp & iitk & iftd \\
\hline ncst & 1667 & 3282 & 3090 & 3086 & 3109 & 47र्ड़्व & 3880 & 3850 \\
\hline iitm & 3230 & 1607 & 4244 & 4280 & 4311 & 5511 & 5204 & 5154 \\
\hline iitb & 3174 & 4336 & 1549 & 4152 & 4199 & 5685 & 5146 & 5054 \\
\hline iisc & 3084 & 4304 & 4098 & 1458 & 4205 & 5657 & 5198 & 5144 \\
\hline doe & 3159 & 4330 & 4088 & 4180 & 1499 & 4665 & 4062 & 3958 \\
\hline iitkgp & 4723 & 6928 & 7048 & 7022 & 6109 & 1582 & 5496 & 6774 \\
\hline iitk & 3908 & 5964 & 5850 & 5986 & 4887 & 4861 & 1498 & 5574 \\
\hline iitd & 3763 & 5792 & 5666 & 5810 & 4641 & 6005 & 5446 & 1542 \\
\hline
\end{tabular}

All values in milliseconds.

Table 9: Predicted Read Operation Timing in DIT Alternative 1.

\begin{tabular}{|c|c|c|c|c|c|c|c|c|}
\hline & ncst & iitm & iitb & ilisc & doe & iitkgp & iitk & iitd \\
\hline ncst & 1667 & 4534 & 4342 & 4338 & 4361 & 6011 & $\overline{5132}$ & 3850 \\
\hline iitm & 5089 & 1607 & 5180 & 5216 & 5247 & 6447 & 6140 & 4231 \\
\hline iitb & 4989 & 5300 & 1549 & 5116 & 5163 & 6649 & 6110 & 4203 \\
\hline iisc & 5079 & 5434 & 5228 & 1458 & 5335 & 6787 & 6328 & 4279 \\
\hline doe & 3893 & 4258 & 4016 & 4108 & 1499 & 4593 & 3990 & 3152 \\
\hline iitkgp & 6709 & 6620 & 6740 & 6714 & 5801 & 1582 & 5188 & 4480 \\
\hline iitk & 5509 & 5948 & 5834 & 5970 & 4871 & 4845 & 1498 & 3957 \\
\hline iitd & 3763 & 4182 & 4056 & 4200 & 3031 & 4395 & 3836 & 1542 \\
\hline
\end{tabular}

All values in milliseconds.

Table 10: Predicted Read Operation Timings in DIT Alternative 2. 


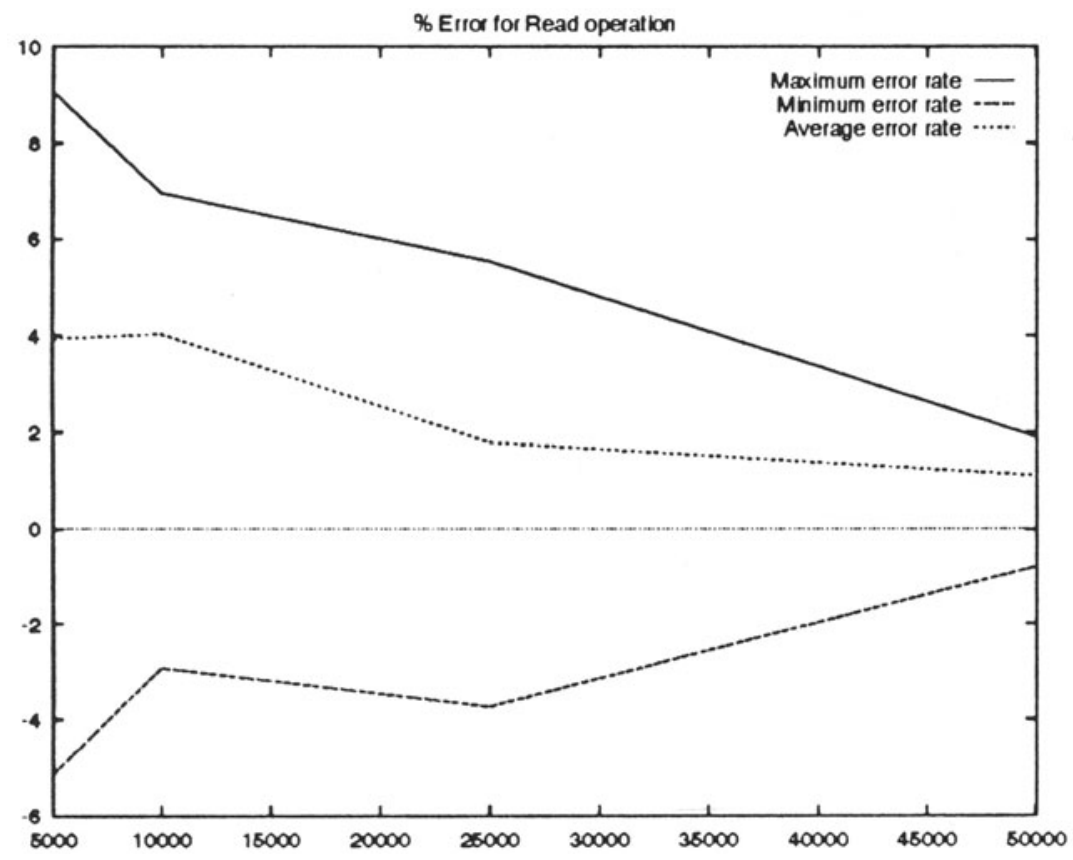

\% Error for Compare operation

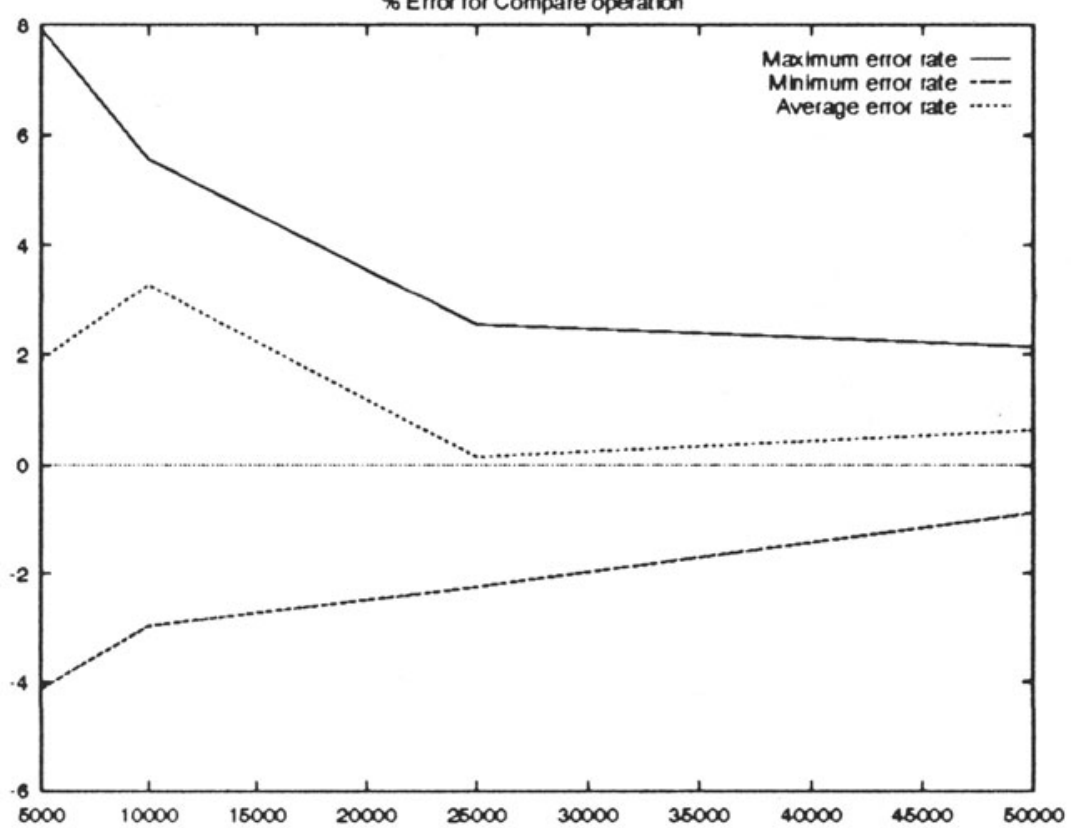



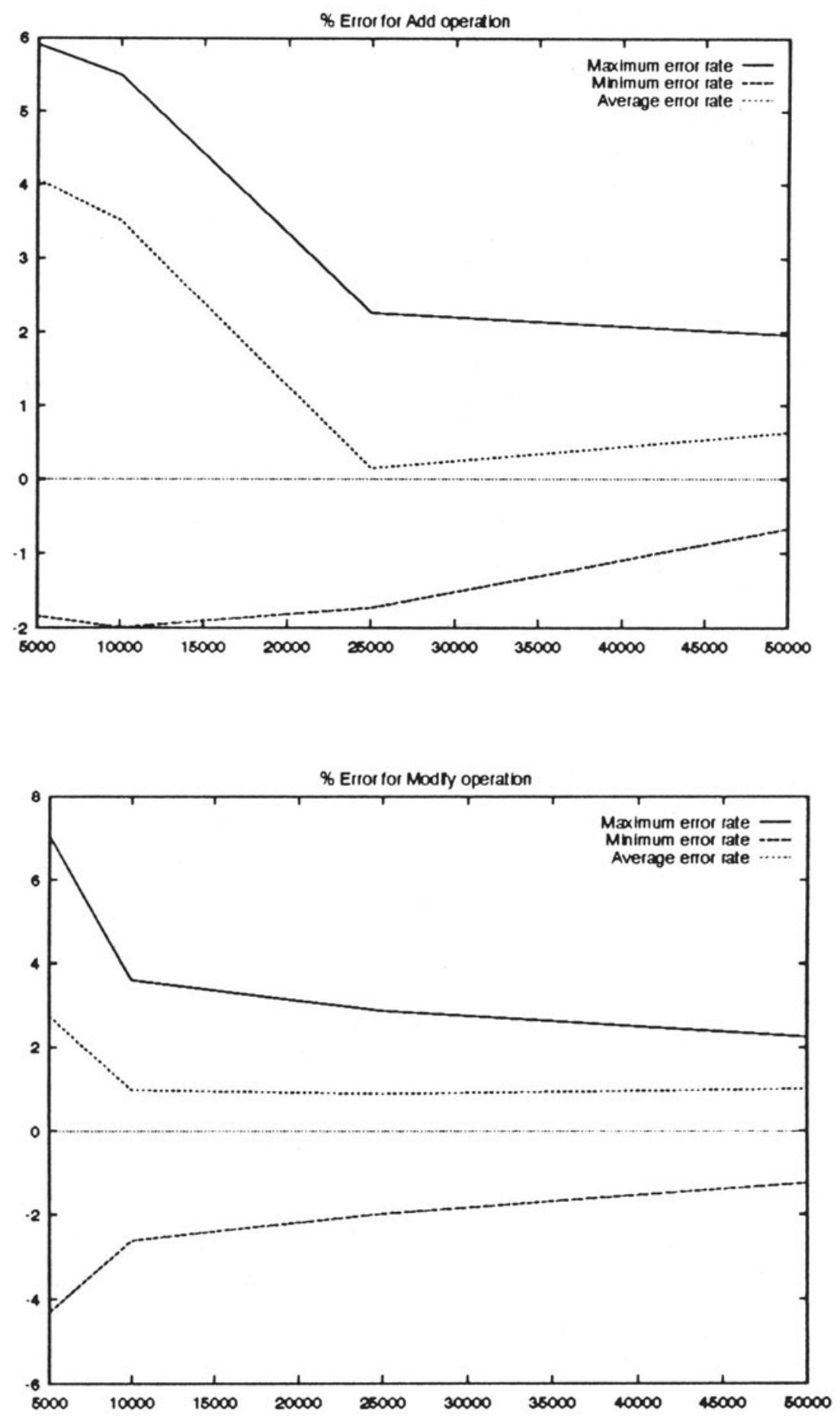

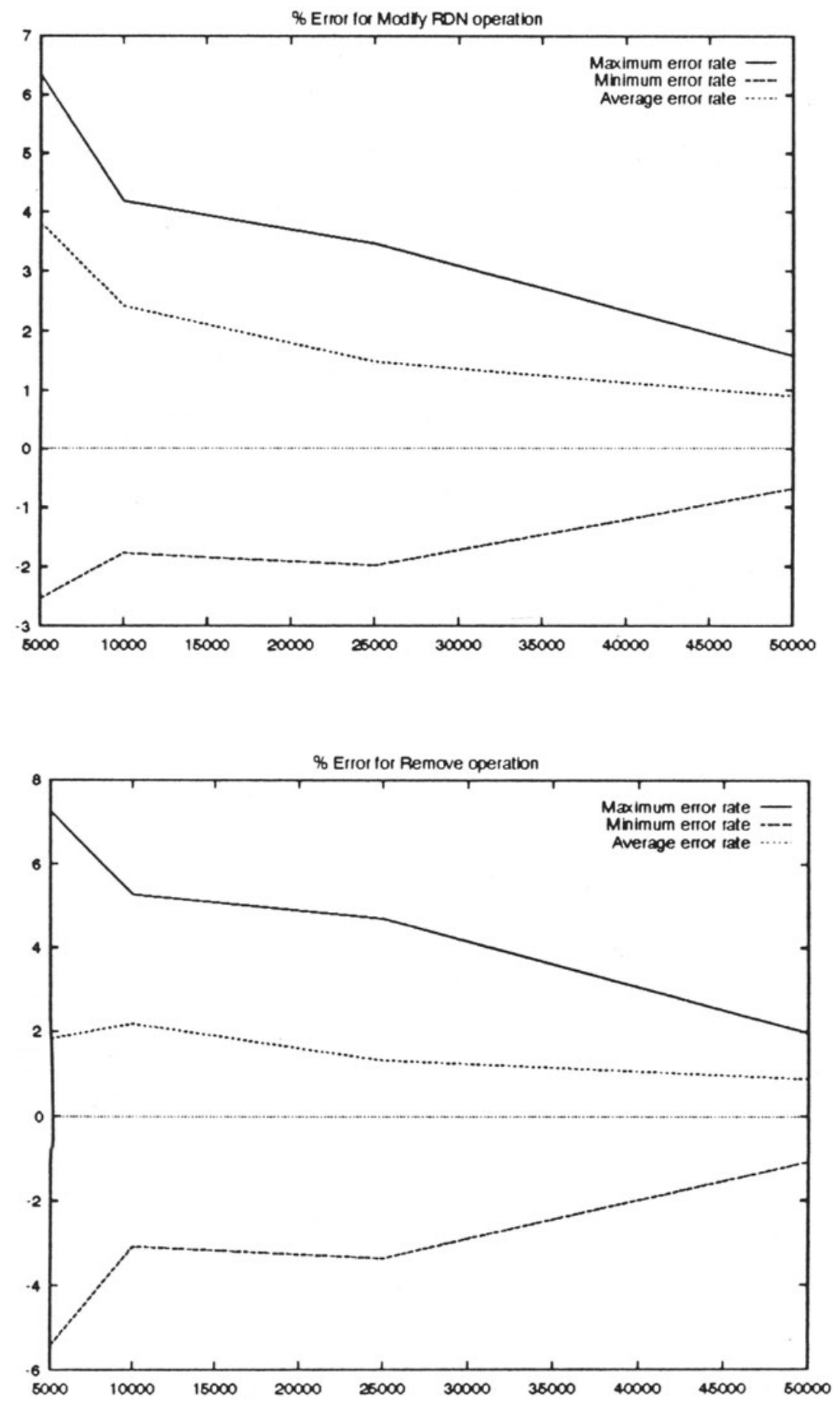

Figure 8: Percentage Error rate Vs Database size, Experiment 1. 

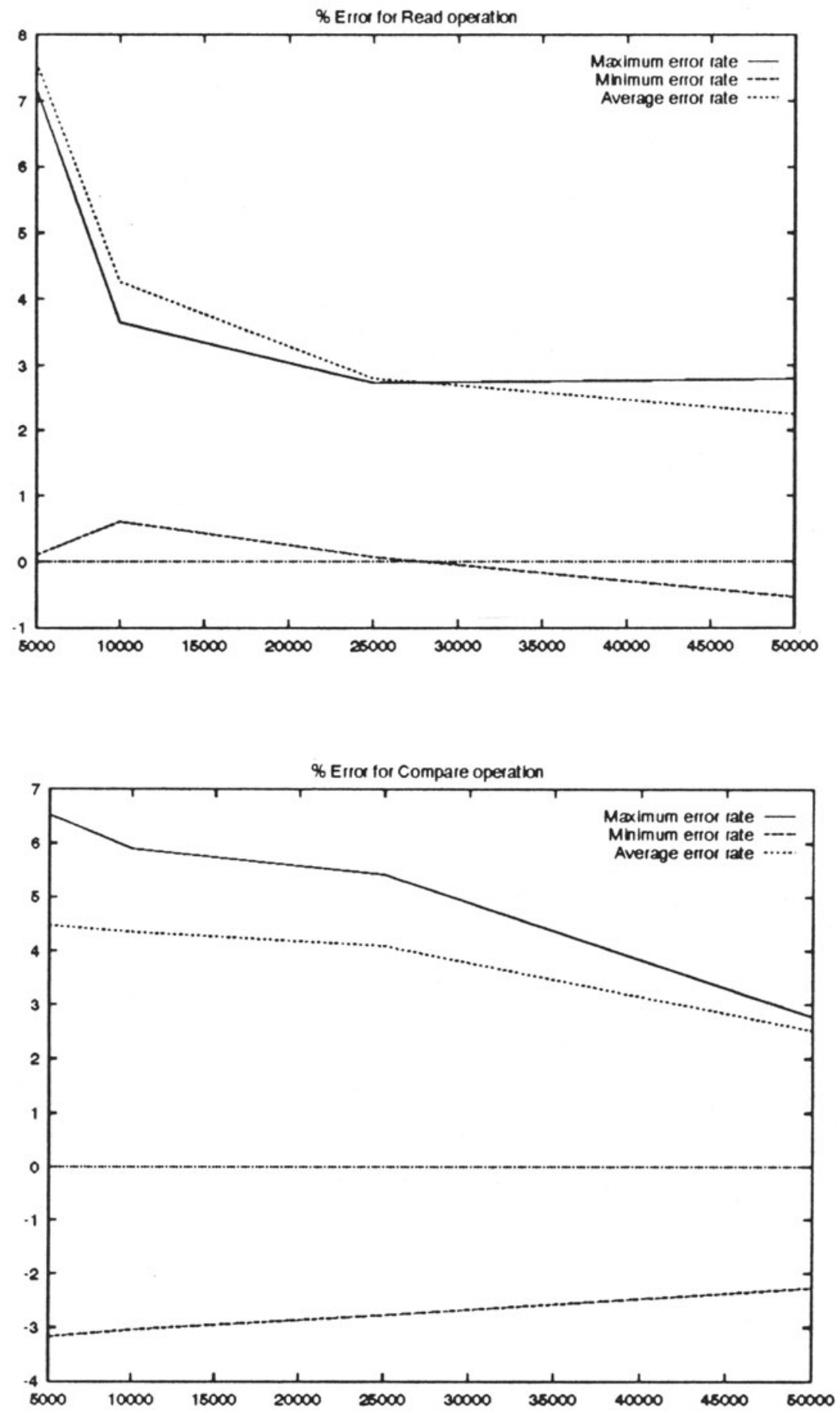

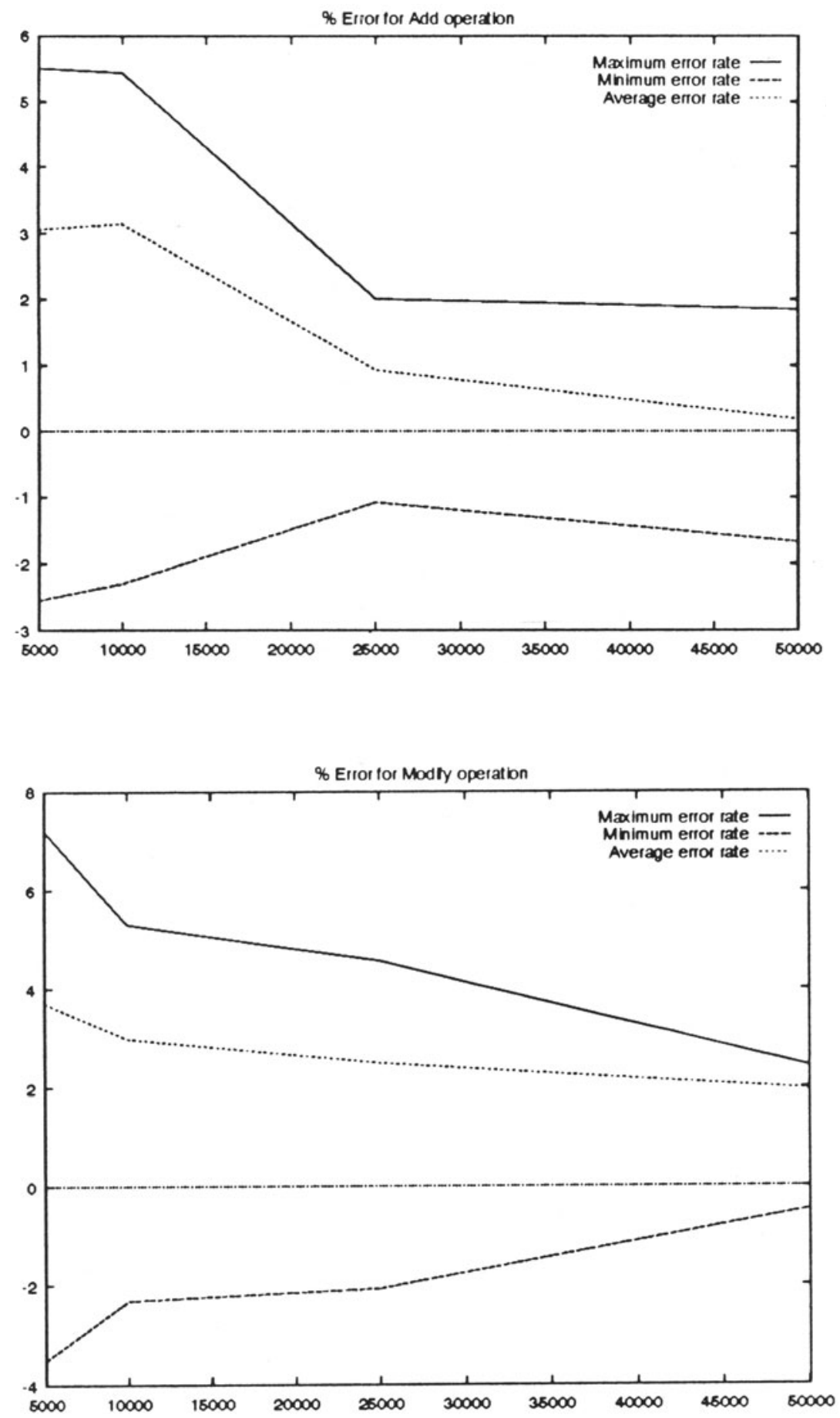

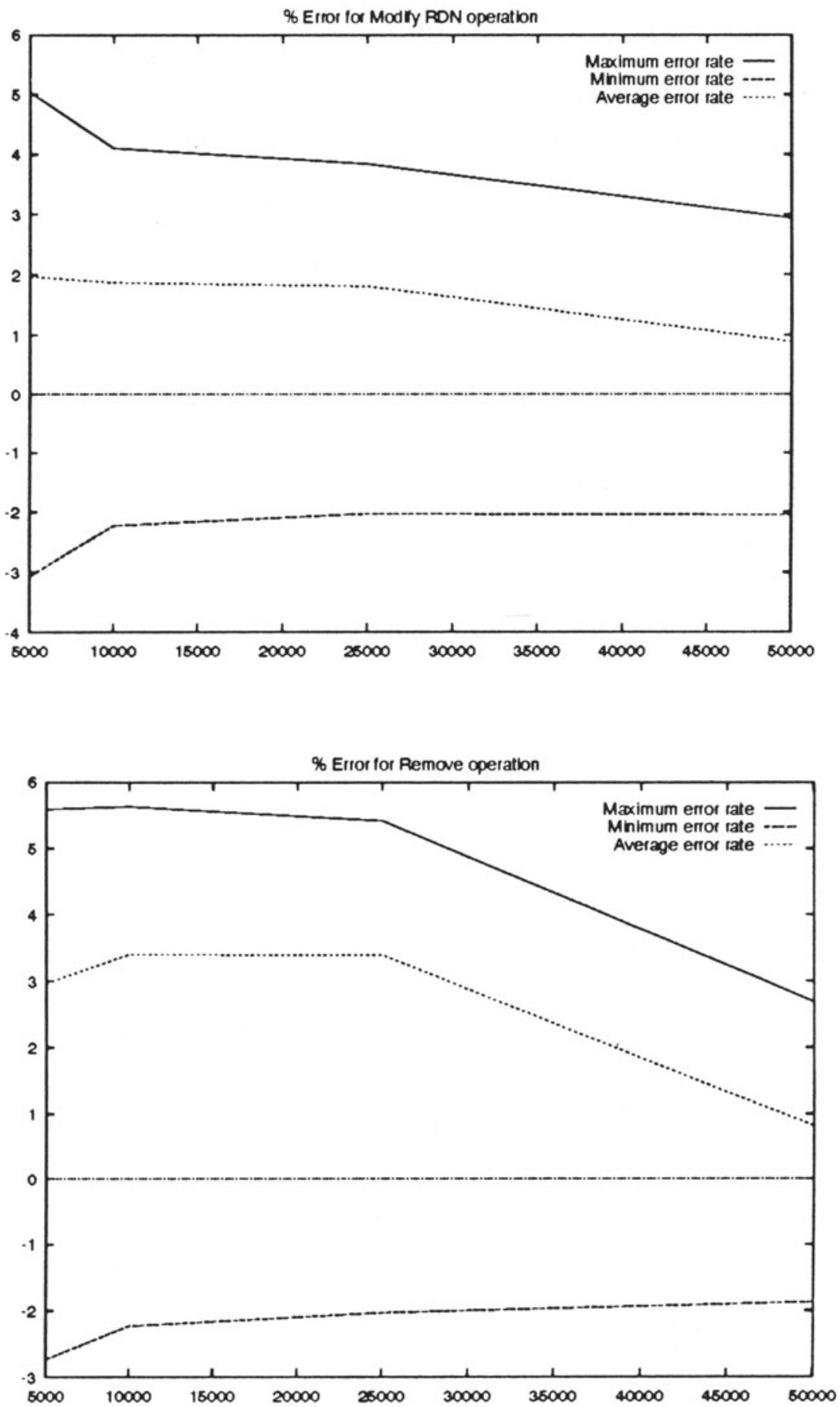

Figure 9: Percentage Error rate Vs Database size, Experiment 2. 

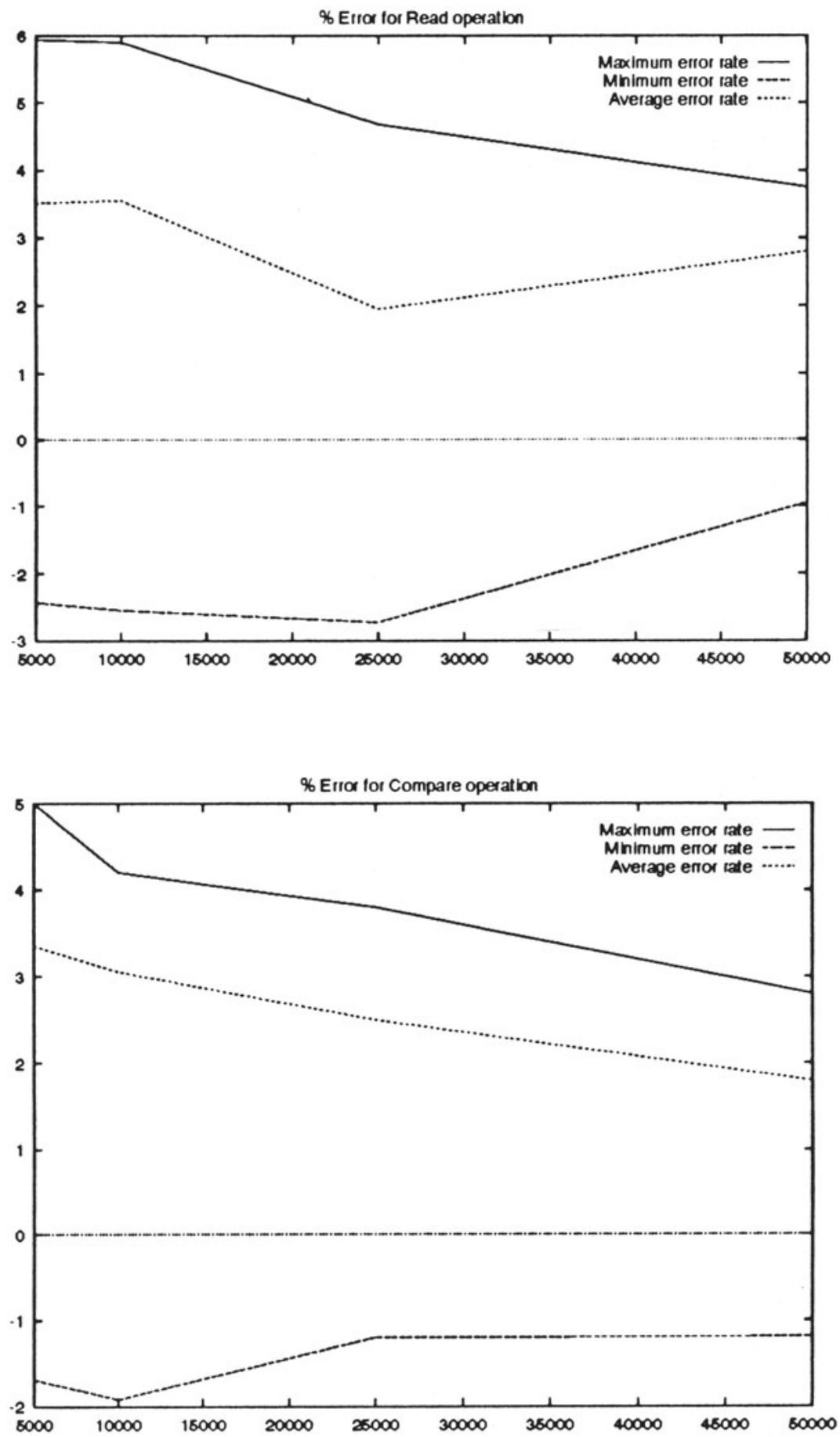

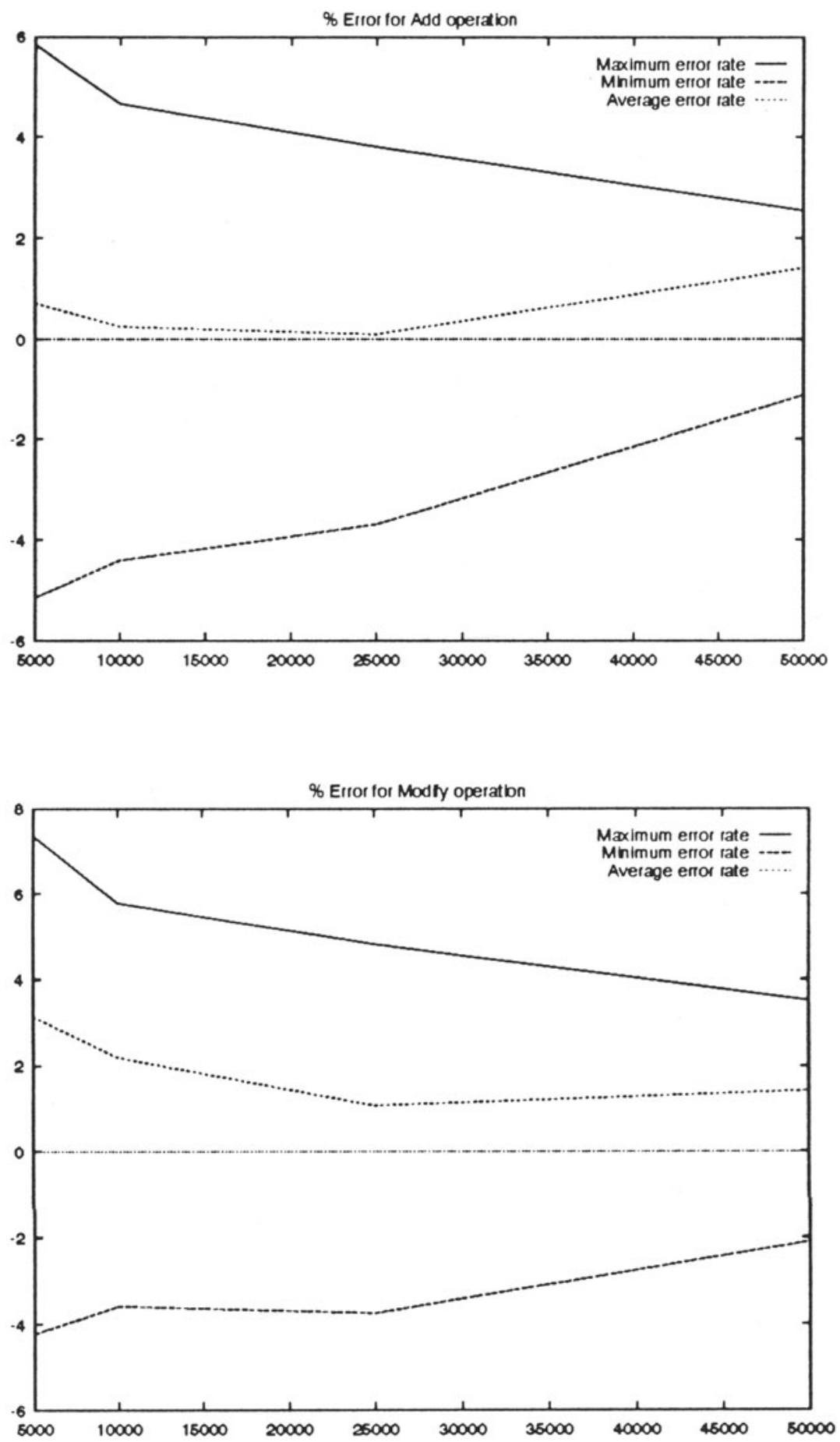

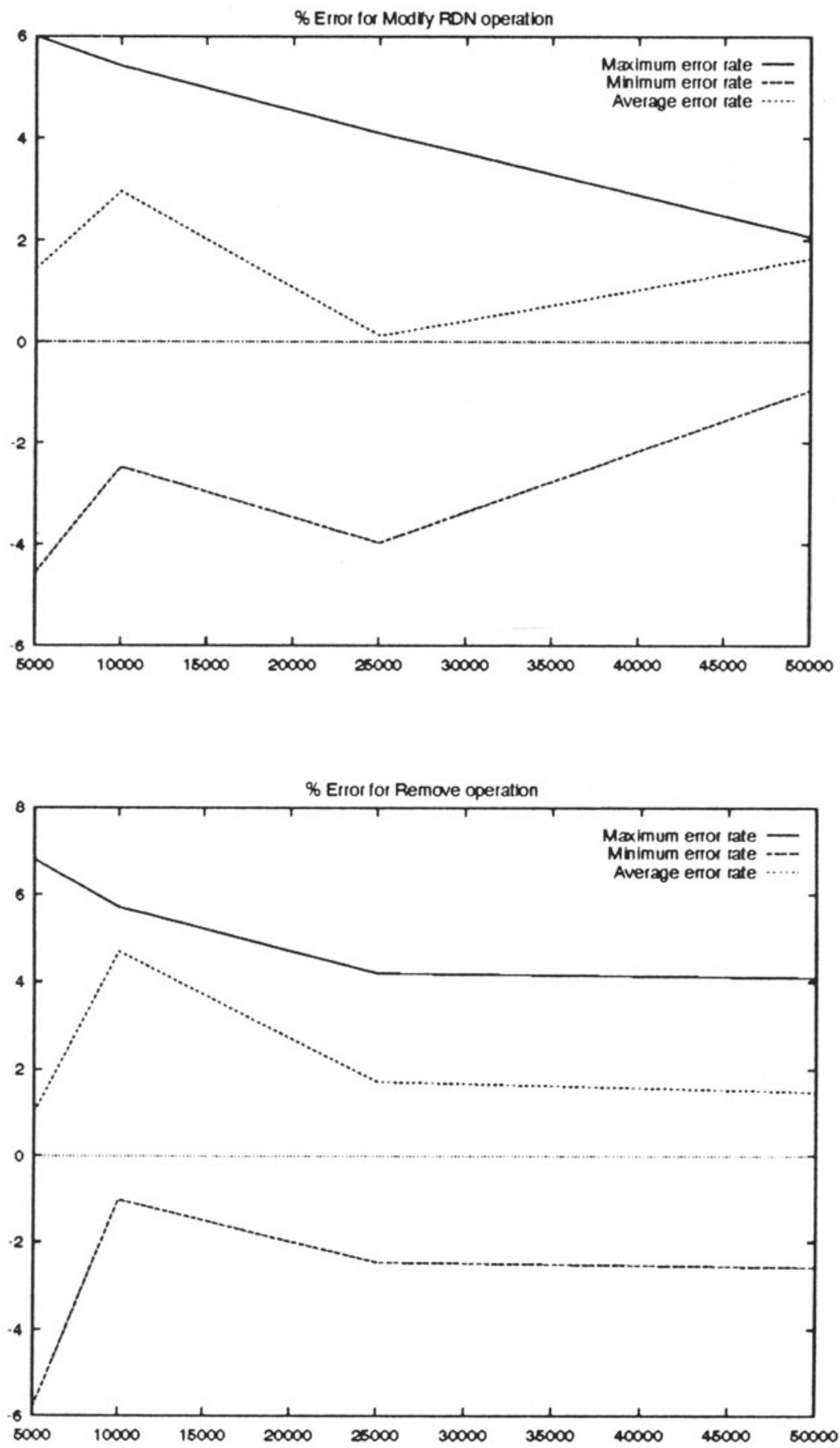

Figure 10: Percentage Error rate Vs Database size, Experiment 3. 

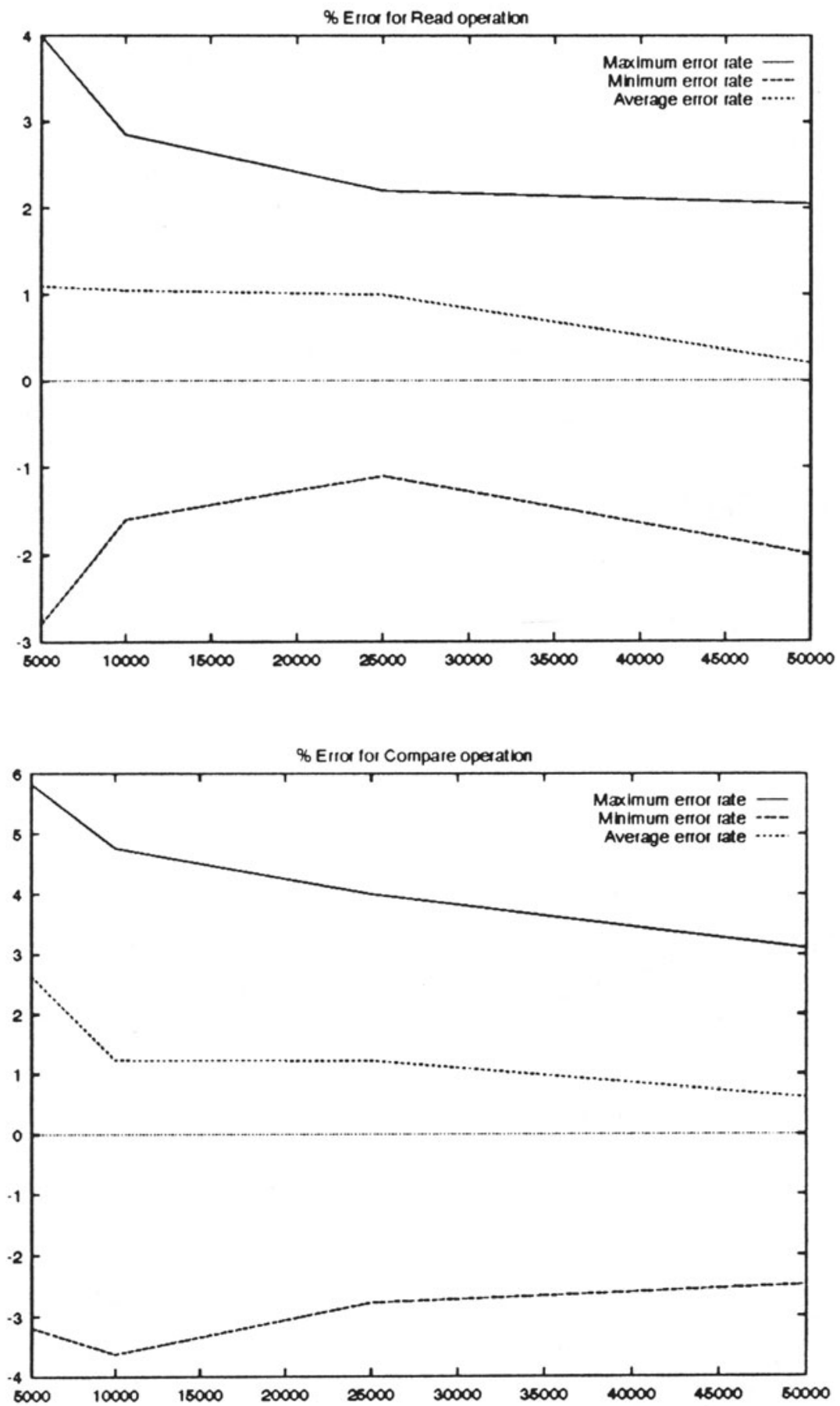

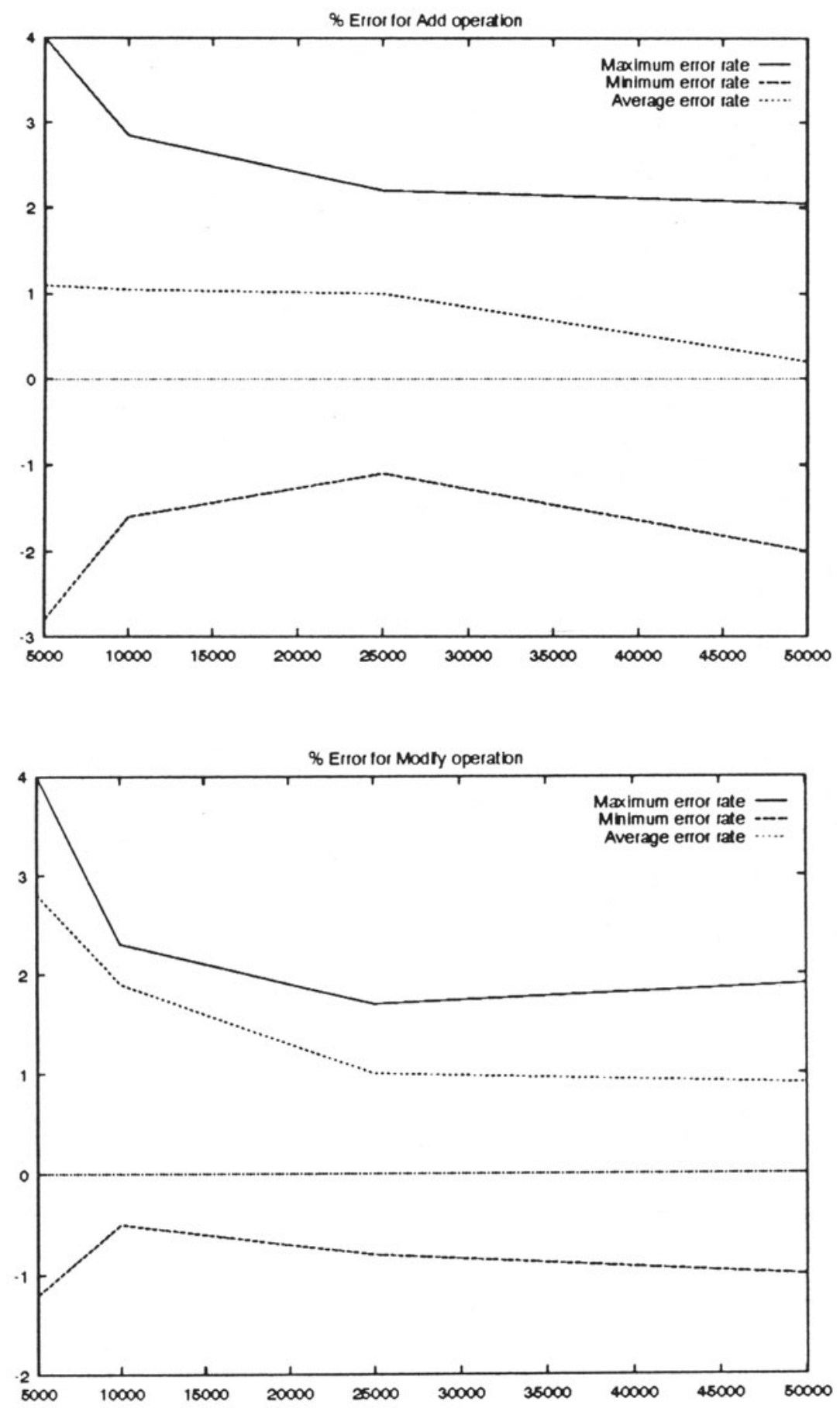

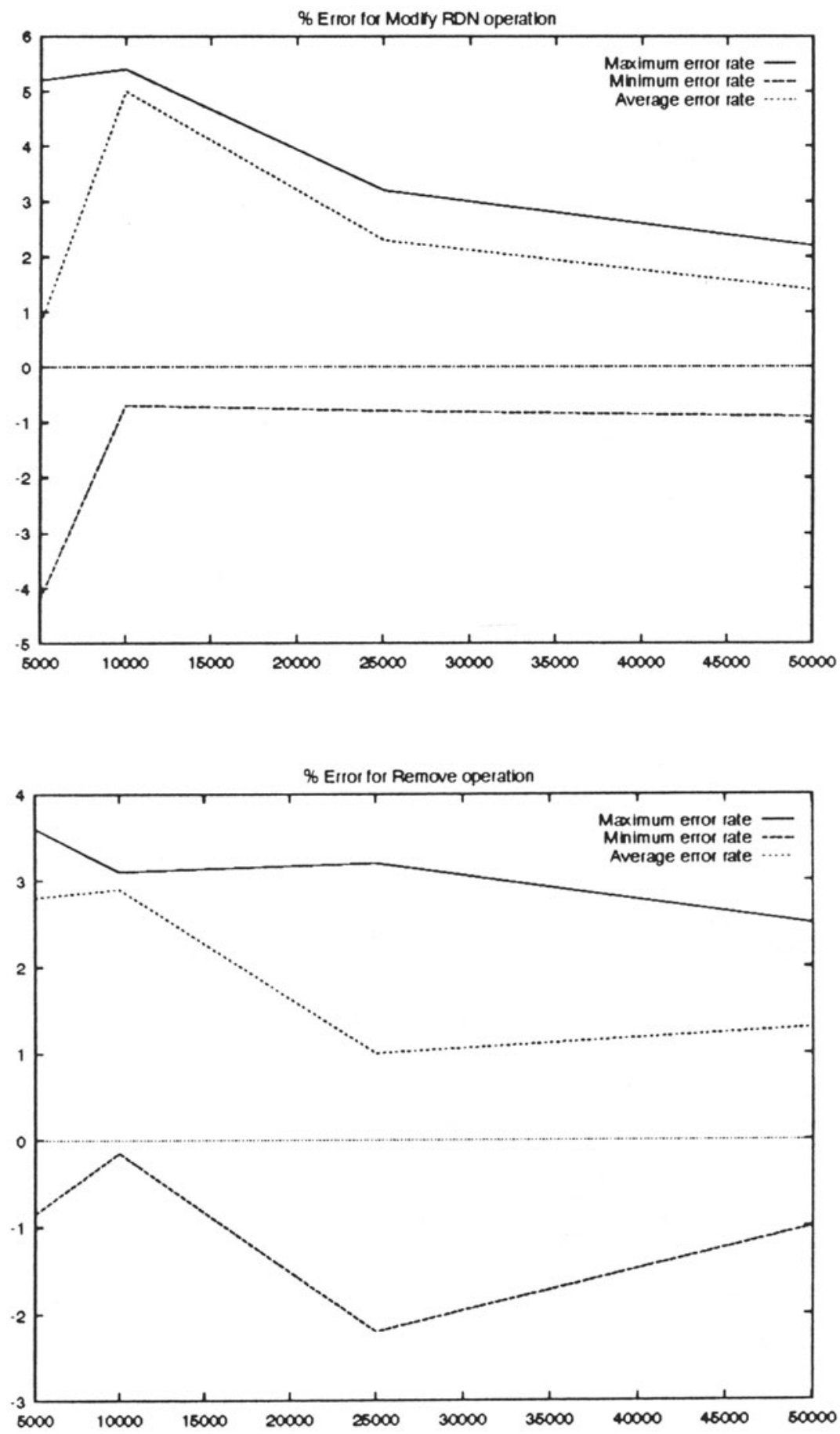

Figure 11: Percentage Error rate Vs Database size, Experiment 4. 